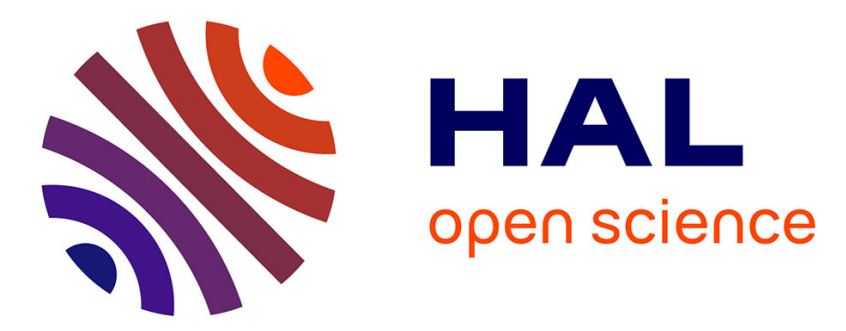

\title{
Interfacial electrokinetic effect on microchannel flow linear stability
}

\author{
Sedat F. Tardu
}

\section{To cite this version:}

Sedat F. Tardu. Interfacial electrokinetic effect on microchannel flow linear stability. Journal of Fluids Engineering, 2004, 126, pp.10-14. 10.1115/1.1637927 . hal-00262003

\section{HAL Id: hal-00262003 \\ https://hal.science/hal-00262003}

Submitted on 18 Feb 2020

HAL is a multi-disciplinary open access archive for the deposit and dissemination of scientific research documents, whether they are published or not. The documents may come from teaching and research institutions in France or abroad, or from public or private research centers.
L'archive ouverte pluridisciplinaire HAL, est destinée au dépôt et à la diffusion de documents scientifiques de niveau recherche, publiés ou non, émanant des établissements d'enseignement et de recherche français ou étrangers, des laboratoires publics ou privés. 


\section{Interfacial Electrokinetic Effect on the Microchannel Flow Linear Stability}

The electrostatic double layer (EDL) effect on the linear hydrodynamic stability of micro-channel flows is investigated. It is shown that the EDL destabilizes the Poiseuille flow considerably. The critical Reynolds number decr eases by a factor five when the nondimensional Debye-Huckel parameter $\kappa$ is ar ound ten. Thus, the transition may be quite rapid for microchannels of a couple of microns heights in particular when the liquid contains a very small number of ions. The EDL effect disappears quickly for $\kappa \geqslant 150$ corr esponding typically to channels of heights $400 \mu \mathrm{m}$ or larger. These $\mathrm{r}$ esults may explain why significantly low critical Reynolds numbers have been encountered in some experiments dealing with microchannel flows.

\section{Introduction}

Both the rapid development of MEMS, and the need for high rates of heat removal of micro electronic devices require a clear understanding of micro flows in channels of heights ranging from some 100 to a couple of $\mu \mathrm{m}$. It is not clear whether the classical macro-approaches are still valid or not in such small devices. Several experimental studies published so far are quite inconsistent, and no clear answer to these questions has been given yet. (Gad-el Hak [1], Tardu [2]).

In liquid flows, the molecular effects inducing slip boundary conditions become important only for microchannels of hydraulic diameters smaller than $1 \mu \mathrm{m}[1,2]$. Thus, it is rather difficult to explain the large departures from conventional transport theory observed in some microchannel flows of larger heights [3,4]. One of the micro-effects that may play an important role is the interfacial effect caused by the electric double layer (EDL) at the solid/ liquid interface. The electrostatic charges present on the solid surface attract the counterions to establish an electrical field. In the compact layer next to the wall and less than $1 \mathrm{~nm}$ thick the ions are immobile. In the diffuse EDL layer however, the ions are less affected by the electrical field and can move. The counterion concentration near the wall is larger than in the bulk of the fluid. That results in a net charge density in a unit volume resulting from the concentration difference between cations and anions, according to the Boltzmann equation. The electrostatic potential at any point near the surface, provided that it is small compared to the energy of ions, may be obtained by a linear approximation of the PoissonBoltzmann equation. Its value at the wall can be related to the Zeta potential between the compact layer and diffuse layer, when the EDLs near the opposite walls do not overlap. The Zeta potential is a property of the solid-liquid pair and can be determined experimentally [5]. The imposed pressure gradient accumulates the mobile ions downstream and sets up an electrical field whose potential is called the streaming or electrokinetic potential. The streaming potential and the net charge density induces a streamwise external force. In the steady state, the streaming current due to the transport of charges is in equilibrium with the conduction current in the opposite direction. That allows the determination of the streaming potential and of the velocity profiles under the EDL effect. In macro scale flows, these effects are negligible, as well as the thickness of the EDL is very small compared to the height of the channel.
The velocity profile under the electrokinetic EDL effect obtained by Mala et al. [6] in the Debye-Hückel approximation, can be put in non-dimensional form as [2];

$$
u=1-y^{2}-4 \frac{I_{1}-I_{2}}{\frac{\kappa^{2} \sinh \kappa}{\bar{\zeta}^{2} G}+4\left(I_{3}-\frac{I_{4}}{\sinh \kappa}\right)}\left\{1-\left|\frac{\sinh \kappa y}{\sinh \kappa}\right|\right\}
$$

where the scaling velocity is the centerline velocity of the Poiseuille component, i.e. $-a^{2} d p / d x / 2 \mu$ and the scaling length is the half channel height $a$. There are several parameters in this equation, for instance $G=\left(n_{0} z e a\right)^{2} / \lambda_{0} \mu$, with $n_{0}$ standing for the ionic number concentration, $z$ for the valence of positive or negative ions, $e$ for the electron charge $\lambda_{0}$ the electric conductivity of the fluid, and $\mu$ for its dynamic viscosity. One of the most important quantities involving in (1) is the non dimensional Debye-Hückle parameter $\kappa=a k=a\left(2 n_{0} z^{2} e^{2} / \varepsilon \varepsilon_{0} k_{b} T\right)^{1 / 2}$ with $\varepsilon$ and $\varepsilon_{0}$ being respectively the dielectric constant of the medium and the permittivity of vacuum, $k_{b}$ the Boltzmann constant and $T$ the absolute temperature. The characteristic EDL thickness is $1 / k$. The non-dimensional Zeta potential reads for $\bar{\zeta}=z e \zeta / k_{b} T$. The quantities $I$ in (1) are given by:

$$
\begin{gathered}
I_{1}=I_{3}=\frac{\cosh \kappa-1}{\kappa}, I_{2}=\left(\frac{1}{\kappa}+\frac{2}{\kappa^{3}}\right) \cosh \kappa-\frac{2}{\kappa^{2}} \sinh \kappa-\frac{2}{\kappa^{3}}, \\
I_{4}=\frac{\sinh \kappa \cosh \kappa}{2 \kappa}-\frac{1}{2}
\end{gathered}
$$

Fig. 1a compares the velocity profile of an "EDL flow" with the conventional Poiseuille flow, when $\kappa=41, G=12720$ and $\bar{\zeta}$ $=2.1254$. This case corresponds to the flow of an infinitely diluted aqueous $\mathrm{KCl}$ solution $\left(n_{0}=3.764 \times 10^{19} \mathrm{~m}^{-3}, \quad \lambda_{0}=7.89\right.$ $\left.\times 10^{-9} \mathrm{~S} / \mathrm{m}\right)$ through a microchannel of height $100 \mu \mathrm{m}$ subjected to a Zeta potential of $50 \mathrm{mV}$. The parameters $G \bar{\zeta}$ and $\kappa$ are exactly those used by Mala et al. [6] who analyzed the electrokinetic effects of the double layer. The Debye length is $1.2 \mu \mathrm{m}$ which is close to the value corresponding to the de-ionized ultrafiltered water experiments of Ren et al. [5] The first impression one has from Fig. 1a is the close similarity between the EDL and Poiseuille profiles with a decrease of the centreline velocity typical to the EDL flows. The important difference however is the presence of an inflexional point at $y \approx 1 / \kappa \arcsin h$ $\left\{-2 / r \kappa^{2} \sinh (\kappa)\right\}$ in the EDL profile where $r$ is the ratio of the 


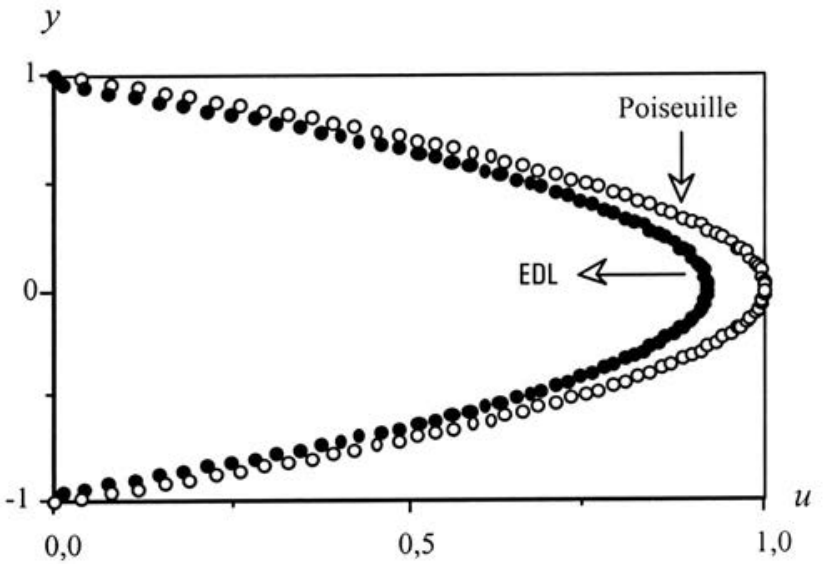

(a)

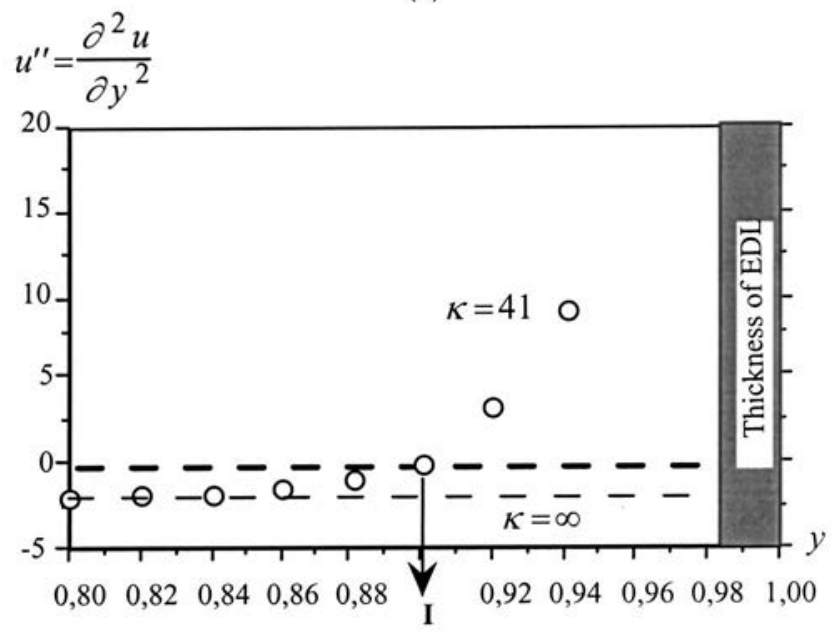

(b)

Fig. 1 EDL and Poiseuille velocity profiles (a), and the inflexional instability of Fjortoft type $(b)$. The parameters of the EDL flow are given in the text. The broken line in (b) corresponds to Poiseuille flow with $\kappa=\infty$ and the circles to the $\kappa=41$ EDL flow. The inflexion point is shown by $\mathrm{l}$. The inviscid instability is of Fjortoft type because after the inflexion point near the wall, $u^{\prime \prime}$ $>0$ and the velocity is smaller than the velocity at the inflexion point $u_{l}$, resulting in $u^{\prime \prime}\left(u-u_{l}\right)<0$ in this zone.

EDL and Poiseuille flows centerline velocities. This makes the flow inviscidly unstable, according to the Fjortoft's criteria.

The linear hydrodynamic stability under the EDL effect is studied through classical methods. The Orr-Sommerfeld equation is solved by a Galerkin-like procedure [7]. The stream function is expanded in a Chebyshev polynomial series with up to 256 terms. The $\tau$-method described by Orszag [8] is used. The disturbances with symmetric streamfunctions are considered only. The eigenvalues of the matrices were numerically computed by the $Q R$ algorithm. The method gave very close results to Grosch and Salwen [9] who used different sets of expansion functions.

Figure 2 shows the neutral curve corresponding to the microchannel-flow with the parameters given below, together with a "macro" Poiseuille flow (i.e. $\bar{\zeta}=0$ or $\kappa \rightarrow \infty$ ). It is clearly seen that the critical Reynolds number decreases by a factor nearly equal to 2 under the effect of EDL: the critical wave and Reynolds numbers of the microflow are respectively $\alpha_{c}=1.10$ and $\operatorname{Re}_{c}$ $=3190$ to be compared with $\alpha_{c}=1.02$ and $\operatorname{Re}_{c}=5772$ of the conventional Poiseuille flow. Due to its inviscid inflexional instability (unstable for $\operatorname{Re} \rightarrow \infty$ for a given $\alpha$ ), the band of unstable wave numbers of the EDL-microflow is significantly larger compared with the Poiseuille-macroflow. The destabilizing EDL effect dis-

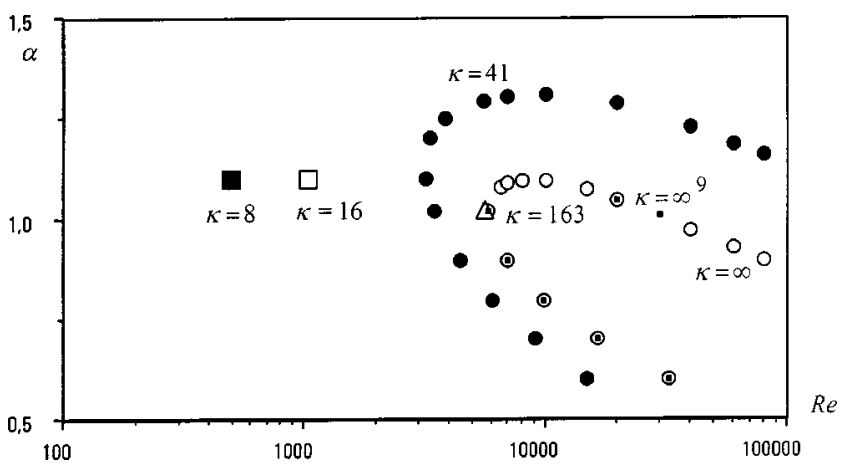

Fig. 2 Neutral curves of the EDL flow compared with the Poiseuille flow. The open circles correspond to Poiseuille flow with $\kappa=\infty$. The results of Grosh and Salwen [9] are shown by small bold squares. Bold circles correspond to $\kappa=41, G$ $=12720$ and $\bar{\zeta}=2.1254$. The rest of the results are obtained by changing the microchannel height and keeping constant the rest of the parameters.

appears quickly when the height of the channel is increased by a factor of $4(400 \mu \mathrm{m})$ (triangle in Fig. 2 corresponding to $\kappa$ $=163$ ). This goes in the same line as previous experimental results showing the lack of micro-effects for the microchannels of height larger than typically $100 \mu \mathrm{m}$. For smaller values of $\kappa$, in return, the effect of the interfacial effects caused by EDL on the transition may be much more severe. For instance, the critical Reynolds number decreases up to $\operatorname{Re}_{c}=1042$ for a channel with a $40 \mu \mathrm{m}$ separation distance subject to the same conditions (the square in Fig. 2 with $\kappa=16$ ), and to a value as small as $\operatorname{Re}_{c}$ $=496$ when $\kappa=8$. This effect, combined with the influence of the roughness that can be critical in microchannel flows, may be the explanation of significantly small transitional Reynolds numbers reported in some studies, and the decrease of $\operatorname{Re}_{c}$ with the decrease of channel size $[3,4]$.

The second parameter that plays an important role in the stability mechanism under the EDL effect is $G \bar{\zeta}^{2}$ (Eq. 1). It is clear that EDL will play a negligible role as $G \bar{\zeta}^{2} \rightarrow 0$, that is, when the Zeta potential becomes very small. Yet, the inflexional instability effect is so strong that the critical Reynolds number is affected even when the electroviscous effects are negligibly small. Figure 3 shows the critical Reynolds number variation versus $G \bar{\zeta}^{2}$ for $\kappa$ $=16$ and $\alpha=1.02$. It is seen that there is a decrease of $30 \%$ in $\operatorname{Re}_{c}$ already at $G \bar{\zeta}^{2}=100$. The EDL and macro-Poiseuille velocity profiles coincide almost perfectly at this low value of $G \bar{\zeta}^{2}$ (not shown here). The second striking feature of Fig. 3 is the rapid decrease of the critical Reynolds number as $G \bar{\zeta}^{2}$ increases, before reaching a plateau region at $G \bar{\zeta}^{2} \approx 10^{4}$.

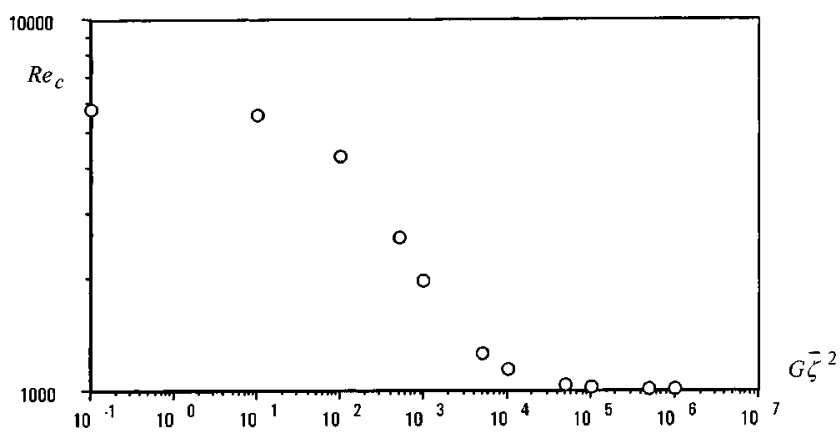

Fig. 3 The critical Reynolds number versus $G \bar{\zeta}^{2}$ for $\kappa=16$ and $\alpha=1.02$. 
The macroscale Poiseuille flow is metastable; i.e., the corresponding stability is subcritical. Nonlinear analysis shows that the instability may occur with finite amplitude when all infinitesimal disturbances are stable [10]. There is a significantly lower critical value of the Reynolds number $\operatorname{Re}_{G}$ compared to $\operatorname{Re}_{c}$, above which the flow is unstable and below which there is no bifurcation. The exact theoretical determination of $\operatorname{Re}_{G}$ is still a matter of research. Experiments show that $\operatorname{Re}_{G} / \operatorname{Re}_{c} \approx 1000 / 5772$ $\approx 1 / 6$. The transitional Reynolds number depends on the shape and shape factors of the channels.

The Poiseuille flow is monotonically stable only for $\operatorname{Re}<100$ to be compared with 5772. The lower limit of the transitional Reynolds number $\mathrm{Re}_{t}$ of channel flows is about 400 according to experiments and the growth rate of three-dimensional disturbances of linear secondary instability [11] (1100 based on the hydraulic diameter and channel averaged velocity). Thus, the ratio $\operatorname{Re}_{t} / \operatorname{Re}_{c}<\frac{1}{15}$ in macroscale Poiseuille flows.

The main non-linear stability mechanism in macro-Poiseuille flow is due to a secondary instability with the development of mean spanwise profiles inflexion with strong shear $[12,13]$. In the EDL flow there is already a streamwise inflexion point in the base flow. Thus, the Reynolds numbers $\operatorname{Re}_{G}$ and $\mathrm{Re}_{t}$ should be much lower under the EDL effect. A nonlinear analysis is necessary to check out this point but the first stage is then the determination of the marginal curve which is done here. The nonlinear saturation of the primary stability and formation of a secondary flow, together with the secondary instability processes have to be analyzed in EDL flow similarly to the Poiseuille macro-flow [11]. Some arguments on the reinforcing effect of the EDL on the subcritical nature of the macro Poiseuille flow may however already be given. The square of the amplitude of a finite disturbance is given by:

$$
\frac{d\left|A_{1}^{2}\right|}{d t}=2 \alpha c_{i}\left|A_{1}\right|^{2}+\left(k_{1}+k_{2}+k_{3}\right)\left|A_{1}\right|^{4}
$$

according to Stuart [14]. The flow reaches a subcritical equilibrium state when $k_{1}+k_{2}+k_{3}>0$. The coefficient $k_{1}$ represents the distortion of the mean motion: it is related to the eigenfunctions of the linear stability problem, and it is negative. The coefficient $k_{2}$ is linked to the generation of the harmonic of the fundamental and is also likely negative. The wall normal distortion of the fundamental $\left(k_{3}\right)$ must "be positive and outweigh the combined negative effect of $k_{1}$ and $k_{2}$ to reach a subcritical state" [14]. Now, $k_{1}$ is proportional to $\operatorname{Re}_{c} \alpha_{c}^{2}$ (Eq. 6.3 of [14]). It has therefore a significantly smaller negative contribution to $k_{1}+k_{2}+k_{3}$ under the EDL effect. Furthermore, part of the terms involving in the coefficient $k_{3}$ is inversely proportional to $\mathrm{Re}_{c}$ according to the Eq. 6.5 of [14] and the EDL presumably reinforces the positive character of $k_{3}$ in the subcritical state.

The experimental verification of the linear stability results is difficult because of the subcritical character of the stability mechanism, and the difficult control of the level of turbulence at the inlet. That also rises the question of the EDL effect on the stability in the developing region. It is also somewhat difficult to reach such high Reynolds numbers in microchannels, even though one may consider the possibility of Debye lengths larger than 1 $\mu \mathrm{m}$, thus higher channels compared with the numerical example given before. Well-controlled experiments are certainly more difficult in microchannels. Yet, it should be possible to detect the EDL effects on the transitional Reynolds number by classical Repressure gradient curves. Table 1 shows the expected transitional Reynolds numbers under the EDL effect as a function of $\kappa$. The ratio $\operatorname{Re}_{t} / \operatorname{Re}_{c}=1 / 15$ is taken same as in macro channels, although, it is more than likely that $\mathrm{Re}_{t} / \mathrm{Re}_{c}$ is presumably smaller under the EDL effect according to the arguments given above. Table 1 also shows the Reynolds numbers achieved in some experiments. It is seen that the expected transitional Reynolds number is within the range of experimental possibilities. For larger channel of typical height $100 \mu \mathrm{m}$ or so, the critical Reynolds number should also be measured, at least in principle. The experi-
Table 1 Estimated values of the transitional numbers under the EDL effect compared with some experiments and the range of Re numbers that can be reached in microchannels. 1: Mala and Li [15], microtubes, the transitional Re number of 150 (second line, last column) correspond to $D_{h} / 2=65 \mu \mathrm{m}$. 2: Gao et al. [18], 2-D microchannels, 3: Qu et al. [19], trapezoidal silicon microchannels. Profiles at $D_{h} / 2-71 \mu \mathrm{m}$ (their Fig. 8) suggest a transitional number of 200. 4: Wang and Peng [4] microchannels. This is a particularly low transitional Reynolds number. The exact value of $D_{h} / 2$ is $150 \mu \mathrm{m}$. The value of $\kappa$ is larger than $\mathbf{1 2 0}$ for other microchannels these authors investigated. Although their experiments have been conducted with distilled water (so that roughly $1 / k=1 \mu \mathrm{m}$ ) the low transitional Reynolds numbers they report for higher channels cannot be explained by the EDL effect. Strong effect of roughness in the inlet region is suspected in these experiments.

\begin{tabular}{|c|c|c|c|c|}
\hline $\begin{array}{c}\text { Channel } \\
\text { height, or } \\
D_{h} / 2(\mu m)\end{array}$ & $\left(\frac{1}{k}=1 \mu m\right)$ & $\begin{array}{l}\text { Critical Reynolds } \\
\text { number } \\
\text { (Transitional } \\
\text { Reynolds number, } \\
\text { estimated) } \\
\operatorname{Re}_{t^{\prime}=\infty}=400\end{array}$ & $\begin{array}{l}\text { Re reached in some } \\
\text { experiments reported in } \\
\text { the literature (Exact } \\
D_{h} / 2 \text { values in } \mu m \text { ) }\end{array}$ & $\begin{array}{c}\text { Reynolds } \\
\text { number in early } \\
\text { transition } \\
\text { reported in } \\
\text { some } \\
\text { investigations }\end{array}$ \\
\hline 100 & 41 & $3190(213)$ & $400^{1}(102.5), 400^{2}(100)$ & $\begin{array}{c}50^{4} \text { (see the } \\
\text { text) } 200^{3 *} \text { (see } \\
\text { the text) }\end{array}$ \\
\hline$\overline{40}$ & 16 & $1042(70)$ & $\begin{array}{c}360^{1}(50.5), 70^{2}(53), \\
95^{3}(33)\end{array}$ & $150^{r^{2}}$ \\
\hline 20 & 8 & $496(33)$ & $250^{1}(25), 23^{3}(23.5)$ & $56-170^{1}$ \\
\hline
\end{tabular}

ments are limited to small Reynolds numbers for microchannels of height smaller than $20 \mu \mathrm{m}$, because very high pressure is then necessary and the channels will then break.

The last column of Table 1 shows transitional Reynolds numbers reported by some authors indicating early transition in microchannel liquid flows. All of these experiments have been conducted with DIUF-W but the channel wall material may be different from one experiment to the other. In lack of experimental details it is hard to give definitive conclusions. Yet, there is a satisfactory agreement between the estimated theoretical $\mathrm{Re}_{t}$ and the measurements, in particular with those of Mala and Li [15]. There is only one value reported by them on which we are sure, but unfortunately other details are not available. They, however, clearly indicated that "there is an early transition from laminar to turbulent flow at $\mathrm{Re}_{t} \geqslant 56-168$ " and that "the range of $\mathrm{Re}_{t}$ values varies somewhat, depending on the diameter and the material of the wall" pointing at a plausible EDL effect. Note that these experiments have been conducted in micro tubes, and that the Poiseuille-Hagen flow is linearly stable. That does not matter for the qualitative comparisons made here, since the nonlinear character of the stability is similar in planar and cylindrical channels.

To conclude, the EDL destabilizes the linear modes of the Poiseuille channel flow and early transition in microchannels is plausible. This effect can be experimentally checked, provided that the liquid contains a very small amount of ions, the Zeta potential is high enough and the channel height is sufficiently small. In practice that would require the use of DIUF-W or organic liquids, and channel heights larger than $20 \mu \mathrm{m}$ (but smaller than typically 100 $\mu \mathrm{m}$ ) for feasibility problems (microchannel failure at high pressures). There is no effect on stability for liquids with high ionic concentration and/or mini channels. The nonlinear stability analysis of the EDL flow is necessary, although physical considerations indicate a much more rapid transition compared to macro flows. Direct Numerical Simulations can also be helpful to this end. Controlled experiments in a way similar to those reported by Ren et al. [5] have to be conducted, by keeping the same channel with the same roughness distribution, and changing the ionic concentration of the liquid. The fact that the channels cross-section shape, in particular the corners have important contribution to the EDL field have also to be considered (Yang et al., $[16,17])$. These results may imply important industrial applications, in particular in the control and manipulation of the heat transfer process in 
microchannels. The complete parametrical study including the EDL effects on amplification and decay rates, and the eigenvalue spectrum is under investigation. The nonlinear stability analysis of EDL flow is also currently investigated.

\section{References}

[1] Gad-el-Hak, M., 1999, "The Fluid Mechanics of Microdevices," J. Fluids Eng., 121, pp. 5-33.

[2] Tardu, S., 2003, "Transferts thermiques dans les microcanaux," Traité EGEM, Tome 6 Ch. 6, Microfluidique, 36p., Hermès.

[3] Peng, X. F., Peterson, G. P., and Wang, B. X., 1994, "Heat Transfer Characteristics of Water Flowing through Microchannels," Exp. Heat Transfer, 7, $265-283$.

[4] Wang, B. X., and Peng, X. F., 1994, "Experimental Investigation on Liquid Forced Convection Heat Transfer through Microchannels," Int. J. Heat Mass Transfer, 37, 73-82.

[5] Ren, L., Qu, W., and Li, D., 2001, "Interfacial Electrokinetic Effects on Liquid Flow in Microchannels," Int. J. Heat Mass Transfer, 44, 3125-3134.

[6] Mala, G. M., Li, D., and Dale, J. D., 1997, "Heat Transfer and Fluid Flow in Microchannels," Int. J. Heat Mass Transfer, 40(13), 3079-3088.

[7] Von Kerczek, C. H., 1982, "The Instability of Oscillatory Poiseuille Flow," J. Fluid Mech., 116, 91-114.

[8] Orszag, S. A., 1971, “Accurate Solution of Orr-Somerfeld Stability Equation," J. Fluid Mech., 50, 689-703.
[9] Grosch, C. E., and Salwen, H., 1968, "The Stability of Steady and Timedependent Plane Poiseuille Flow," J. Fluid Mech., 34, pp. 177-205.

[10] Drazin, P. G., and Reid, W. H., 1981, Hydrodynamic Stability, Cambridge University Press, Cambridge, UK, pp. 370-464.

[11] Orszag, S. A., and Patera, A. T., 1983, "Secondary Instability of Wall-Bounded Shear Flows," J. Fluid Mech., 128, pp. 347-385.

[12] Henningson, D. S., and Kim, J., 1991, "On Turbulent Spots in Plane Poiseuille Flow," J. Fluid Mech., 228, pp. 183-205.

[13] Itoh, N., 1974, "Spatial Growth of Finite Wave Disturbances in Parallel and Nearly Parallel Flows. Part 1: The Theoretical Analysis and the Numerical Results for Plane Poiseuille Flow," Transactions of the Japan Society of Aeronautics and Space Sciences, 17, 160-174.

[14] Stuart, J. T., 1960, "On the Nonlinear Mechanics of Wave Disturbances in Stable and Unstable Parallel Flows. Part 1: The Basic Behavior in Plane Poiseuille Flow," J. Fluid Mech., 9, 353-370.

[15] Mala, G. M., and Li, D., 1999, "Flow Characteristics of Water in Microtubes," Int. J. Heat Fluid Flow, 20, 142-148.

[16] Yang, C., and Li, D., 1997, "Electrokinetic Effects on Pressure-driven Liquid Flows in Rectangular Microchannels," J. Colloid Interface Sci., 194, 95-107.

[17] Yang, C., and Li, D., 1998, "Analysis of Electrokinetic Effects on the Liquid Flow in Rectangular Microchannels," Colloids Surf., 143, 339-353.

[18] Gao, P., Le Person, S., and Favre Marinet, M., 2002, "Scale effects on Hydrodynamics and Heat Transfer in Two-Dimensional Mini and Microchannels," Int. J. Therm. Sci., 41, p. 10.

[19] Qu, W., Mala, G. M., Li, D., 2000, "Pressure-driven Water Flows in Trapezoidal Silicon Microchannels," Int. J. Heat Mass Transfer, 43, 353-364. 